\title{
Novel biosensors based on screen printed electrode supported with RuO2@graphene nanoribbons composite: NADH and ethanol sensing
}

\author{
$\underline{\text { Dalibor M. Stanković }}^{1,2^{*}}$ Vesna Vukojević $^{2}$, Sladjana Djurdjić ${ }^{2}$, Aleksandar Vukadinović ${ }^{1}$, Miloš \\ Ognjanović ${ }^{1}$, Kurt Kalcher ${ }^{3}$, Bratislav Antić ${ }^{1}$ \\ ${ }^{1}$ The "Vinča" Institute of Nuclear Sciences, University of Belgrade, POB 522, 11001 Belgrade, Serbia \\ ${ }^{2}$ Innovation Center of the Faculty of Chemistry, University of Belgrade, Studentski trg 12-16, 11000 \\ Belgrade, Serbia \\ ${ }^{3}$ Institute of Chemistry - Analytical Chemistry, Karl-Franzens University Graz, A-8010 Graz, Austria \\ Corresponding author: dalibors@chem.bg.ac.rs daliborstankovic@vin.bg.ac.rs
}

\begin{abstract}
:
In this work we proposed electrochemical ethanol and NADH biosensor based on screen printed electrode modified with $\mathrm{RuO}_{2} @ g$ graphene nanoribbons composite. Additionally, surface was modified with alcohol dehydrogenase for the preparation of amperometric biosensor for ethanol analysis. Used material was synthesized and characterized using several microstructural (scanning electron microscopy, FTIR, XRD) and electrochemical techniques (CV, EIS). Electrochemical response of tested analyte was investigated as a function of important parameters such as $\mathrm{pH}$, applied working potential, electrode preparation. Under optimal conditions working linear range and limit of detection for ethanol sensing was found to be $1-1800 \mu \mathrm{M}$ and $0.52 \mu \mathrm{M}$, respectively. Moreover, effect of some possible interfering compounds was investigated and developed procedure was tested for application in real samples.
\end{abstract}

Key words: ethanol biosensor; screen printed electrode; graphene nanoribbons, alcohol dehydrogenase;

\section{Introduction}

In the last few decades, significant efforts have been made to develop various biosensors for the detection of numerous biological compounds such as ethanol, glucose, amino acids [1]. Aldo many of these compounds can be quantified with several methods, including chromatography and spectrophotometry, greatest concern is dedicated to electrochemical biosensors. Electrochemical biosensors are preferable due to their low-cost, ease of manipulate, relatively fast response times and small size. Carbon nano-materials, such as SWCNT, MWCNT, graphene and recently graphene nanoribbons, are very popular in biosensing as they have several good properties such as high surface area, acceptable biocompatibility, chemical and electrochemical stability and good electrical conductivity [2].

\section{Experimental}

\section{Synthesis nanomaterial of Format of your Paper}

In order to get $\mathrm{RuO}_{2}$-GNR/SPCE working electrode, firstly we perform synthesis of $\mathrm{RuO}_{2} / \mathrm{GNR}$ composite, following the procedure described in literature [3]. Briefly, $5 \mathrm{mg}$ of graphene nanoribbons were suspended in 10 $\mathrm{ml}$ of ultra-pure water and this mixture was sonicated for 1 hour. Then, in this suspension $311 \mathrm{mg}$ of $\mathrm{RuCl}_{3}{ }^{*} \mathrm{XH}_{2} \mathrm{O}$ was added, with continuous stirring. Potassium hydroxide $(0.1 \mathrm{M})$ was used during stirring in order to obtain $\mathrm{pH}$ values at 7 . After $12 \mathrm{~h}$, solution was centrifuged and washed three times with ultra-pure water and one time with ethanol. Prior use, GNR$\mathrm{RuO}_{2}$ particles was dried, dissolved in DMF (concentration of solution was $5 \mathrm{mg} / \mathrm{ml}$ ) and sonicated for 3 hours. After this period, $5 \mu \mathrm{L}$ of obtained composite were deposit on SPCE electrode and allowed to dry at room temperature. In described way, we have obtained GNR-RuO 2 /SPCE modified electrode. 


\section{Results and discussion}

Electrochemical characterization of modified electrode was performed in $5 \mathrm{mM}\left[\mathrm{Fe}(\mathrm{CN})_{6}\right]^{4-3-}$ prepared in $0.1 \mathrm{M}$ phosphate buffer solution $(\mathrm{pH}$ 7.50) with a scan rate of $50 \mathrm{mV} / \mathrm{s}$. Obtained voltammograms are present at Figure 1. As can be seen, with SPCE as working electrode, neither oxidation or reduction peak do not appear. In case of all others examined electrodes, both peaks were present. The highest current and the best peak shape was obtained using $\mathrm{RuO}_{2}$-GNR/SPCE electrode, which confirm that nanoparticles of $\mathrm{RuO}_{2}$ in cooperation with GNR, significantly improve the characteristics of bare electrode. SEM image of synthesized material was given in Figure 2.

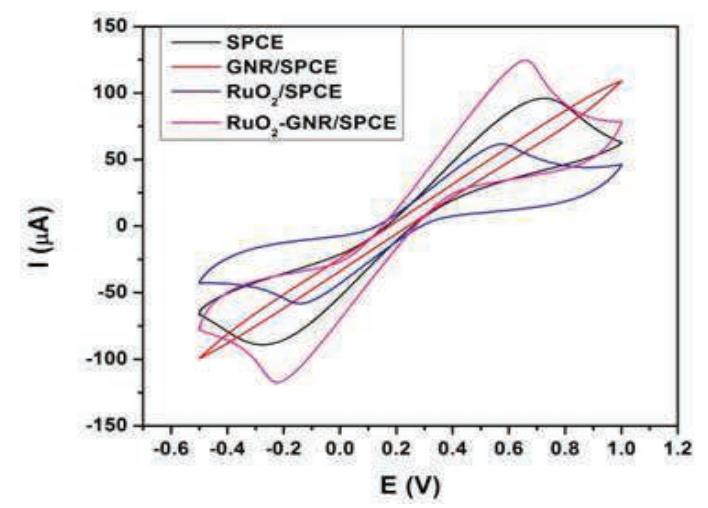

Fig. 1. Cyclic voltammograms performed in $5 \mathrm{mM}$ [Fe(CN)6]4-/3- prepared in 0.1M phosphate buffer solution ( $\mathrm{pH}$ 7.50) with SPCE, GNR/SPCE, RuO2/SPCE and RuO2-GNR/SPCE with a scan rate of $50 \mathrm{mV} / \mathrm{s}$.

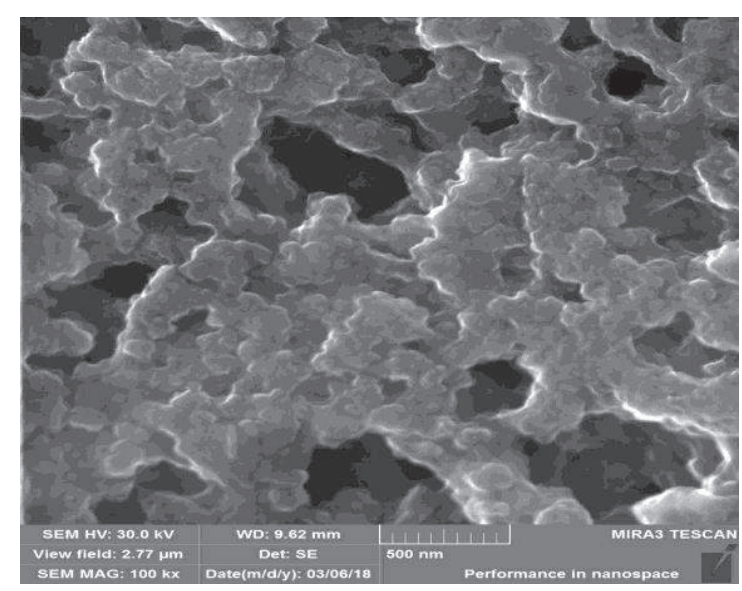

Fig. 2. SEM image of RuO2-GNR

[1] G. Maduraiveeran, M. Sasidharan, V. Ganesan, Electrochemical sensor and biosensor platforms based on advanced nanomaterials for biological and biomedical applications, Biosensors \& bioelectronics 103 113-129(2018); doi: 10.1016/j.bios.2017.12.031

[2] S. Gupta, C.N. Murthy, C.R. Prabha, Recent advances in carbon nanotube based electrochemical biosensors, International journal

\section{Analytical possibilities for ethanol detection}

Under previously optimized experimental conditions, amperometric response for detection of different concentration of ethanol was tested with developed biosensor. Used potential was $0.6 \mathrm{~V}$. It was found that proposed electrode has wide linear range from 1 to 1800 $\mu \mathrm{M}$, with a limit of detection of $0.52 \mu \mathrm{M}$. reproducibility of the 5 measurements of ethanol at the concentrations of 5, 100 and 500 $\mu \mathrm{M}$ was found to be $4.1 \%, 3.1 \%$ and $2.7 \%$, respectively. In addition, operating stability of the biosensor was tested by daily measurements of $150 \mu \mathrm{M}$ of ethanol during 5 days. Decreasing of the response up to the 67 $\%$ was noticed. Amperometric response of ethanol was given in Figure 3.

Application of the developed biosensor for estimation of ethanol content in real samples was done. Results in good agreement with standard method and recovery tests show excellent correlation, indicating high precision and accuracy of the proposed method.

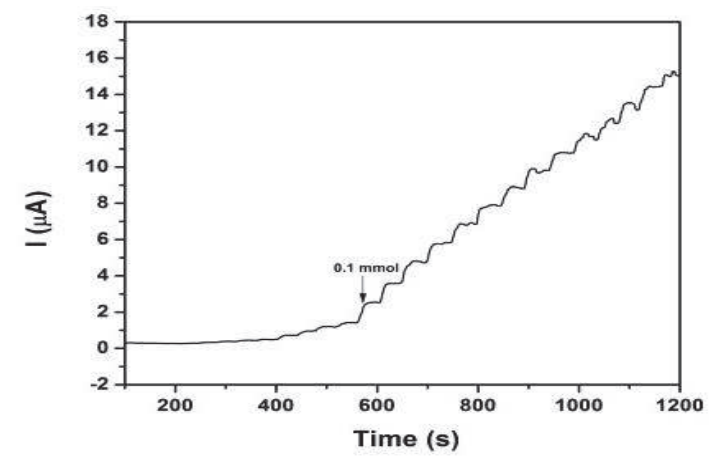

Fig.3. Chronoamperometric responses of the ethanol

of biological macromolecules 108 687-703 (2018) doi: 10.1016/j.ijbiomac.2017.12.038

[3] F.Z. Amir, V.H. Pham, J.H. Dickerson, Facile synthesis of ultra-small ruthenium oxide nanoparticles anchored on reduced graphene oxide nanosheets for high-performance supercapacitors, RSC Adv. 5 67638-67645 (2015) doi:10.1039/C5RA11772K 